\title{
How Universal is the Use of Facemask During COVID-19 Pandemic? A Photo-Epidemiology Study from the Indian State of Kerala
}

\begin{abstract}
Anand Marthanda Pillai ${ }^{1,2}$, Shaffi Fazaludeen Koya ${ }^{1,3 *}$, Mini GK ${ }^{1}$, Jinbert A Lordson ${ }^{1}$, Grace A Chitra ${ }^{1}$, Minu Abraham ${ }^{1}$, Vinod VG Kumar ${ }^{1}$, Sandhya Bijumone ${ }^{1}$, Bindhya Vijayan ${ }^{1}$, Deepu KV ${ }^{1}$, Krishna SH${ }^{1}$, Soji D Jose ${ }^{1}$, Rinsa Vaheed ${ }^{1}$, Revu J ${ }^{1}$, Archana $K^{1}$, Salman R Khan ${ }^{1}$, Sunitha SB ${ }^{1}$, Greeshma J ${ }^{1}$, Shringa Sivanand ${ }^{1}$, Kapila VS ${ }^{1}$, Nissy VL ${ }^{1}$, Amrutha B $^{1}$, Mithun TK ${ }^{1}$, Anjana VM ${ }^{1}$, Grace Wilson ${ }^{1}$, Lal Sreemathy Sadasivan ${ }^{1}$, Kesavan Rajasekharan Nayar ${ }^{1}$ and A Marthanda Pillai $^{1,2}$

${ }^{1}$ Global Institute of Public Health, Thiruvananthapuram, Kerala, India

${ }^{2}$ Ananthapuri Hospitals and Research Institute, Thiruvananthapuram, Kerala, India

${ }^{3}$ Boston University School of Public Health, Boston, Massachusetts, USA

*Corresponding author: Shaffi Fazaludeen Koya, Global Institute of Public Health, Thiruvananthapuram, Kerala, India and Boston University School of Public Health, Boston, Massachusetts, USA
\end{abstract}

\section{ARTICLE INFO}

Received: 幽 April 22, 2021

Published: May 03, 2021

Citation: Anand Marthanda P, Shaffi Fazaludeen K, Mini GK, Jinbert AL, Grace AC, et al., How Universal is the Use of Facemask During COVID-19 Pandemic? A Photo-Epidemiology Study from the Indian State of Kerala. Biomed J Sci \& Tech Res 35(3)2021. BJSTR. MS.ID.005712.

Keywords: COVID 19; Face mask; India; Kerala; Pandemic; Photo Epidemiology

\section{ABSTRACT}

Background \& Objectives: Kerala state was the first to report COVID-19 case in India and was initially successful in curbing the spread of the virus. However, Kerala witnessed a surge of cases subsequently. This study aimed to estimate the prevalence of face mask use and social distancing in public places in Kerala.

Methods: We did a cross-sectional photo-epidemiology study; wherein photographs of people in public places were taken across Kerala. Photos were analysed to determine the usage of mask, whether it was properly worn and the type of mask. Crowding was taken as a surrogate for social distancing. We compared mask use and crowding across district zones with low, medium and high COVID-19 burden.

Results: We analysed 1445 photos capturing 4500 people with clear view of the face. Prevalence of mask use was $96 \%$ across the state. We found near complete mask use in the districts with high COVID-19 burden (99\%) compared to districts with low (96\%) and medium burden (95\%). $20 \%$ of the people in public spaces either did not wear a mask or wore it improperly. Crowding was higher in high burden districts with a weak but positive correlation with case burden.

Conclusion: Prevalence of face mask use was high across all districts in the Kerala State, but a considerable number of people were unprotected due to improper or non-usage of masks. Crowding in high burden districts indicate that inadequate social distancing could have contributed to increased cases, despite a high compliance to mask usage even in these districts.

\section{Introduction}

The state of Kerala (population 34 million) was the first to report the Corona Virus Disease 2019 (COVID-19) case in India on January 30, 2020 in three medical students who returned from Wuhan, China [1]. Prompt quarantine measures effectively stopped transmission from these cases. The state government initiated a series of public health measures including lockdown, 
social distancing and mandatory wearing of facemasks $[2,3]$. The next wave of cases was reported subsequent to the return of nonresident Keralites from the gulf countries and Europe. This wave of infection was contained to double digits by May 2020, with no case of infection reported for nearly 5 days in the first week of May [4]. However, with the repatriation flights and interstate travel in June, the next wave was reported. This surpassed the previous wave of transmission with daily reported cases in the state surpassing 3000 and subsequent declaration of community transmission in the state. Despite aggressive epidemic control measures which included testing, contact tracing and quarantine supported by a very strong grassroot primary health care network and public health measures, Kerala continued to see rising cases of infection six months into the epidemic. Of the fourteen administrative units called districts, Thiruvananthapuram (the capital city) followed by Malappuram reported the highest daily and cumulative number of cases during August. The state reported 66761 cases as on August 28, 2020 (1964 cases/million population).

Universal face mask usage is considered as one of the key strategies to control the spread of COVID-19 in addition to hand washing, social distancing and avoiding closed spaces [5-7]. The role of mask was initially thought to be for source control; recommended for patients with upper respiratory symptoms to prevent spread of the virus from them [8]. Furthermore, asymptomatic and presymptomatic patients were also found to have viral shedding equivalent to symptomatic patients [9] with risk of transmission starting a few days before symptom onset and infectivity peaking at the time of symptom onset for the Severe acute respiratory syndrome coronavirus (SARS CoV-2) [10]. This mandated universal mask use particularly, in places with potency for community transmission and high risk of viral spread. In addition to preventing transmission from infected patients, masks protect the wearer from becoming infected by blocking viral particles from entering the nose and the mouth [11]. The type of mask and the technique of mask usage is key to ensuring effectiveness of universal masking [12]. Although the cloth mask is inferior to the surgical mask or the N95 masks in terms of virus filtering capacity, in a low transmissionrisk setting such as in public places, cloth masks are thought to be sufficient [13].

If infection happens despite mask usage, masks are thought to reduce the inoculum of the virus which may lead to asymptomatic or only mild infection [14]. The usage of masks has been commonly noted to be improper with inadequate coverage of the nose or sometimes with full exposure of the nose and mouth with the mask being pulled down to the neck which makes the mask usage ineffective [15]. This study aimed to estimate the prevalence of face mask usage amongst the public in Kerala during the COVID-19 pandemic using photo-epidemiology. Photo epidemiology is a simple and effective method to study the mask usage and social distancing. Mobile phone cameras can be used to capture photos in public setting with relatively less intrusion into privacy $[11,16]$. Specifically, the study tried to estimate the extent of mask use across the districts of Kerala, the urban-rural and gender difference variations. We counted faces per photo frame and used it as an indicator of crowding and social distancing. We also tried to correlate the COVID-19 case load at the district level with mask use and crowding.

\section{Material \& Methods}

We used a cross-sectional design and obtained photos in public spaces across all the 14 districts of Kerala. Master of Public Health students at the Global Institute of Public Health, Thiruvananthapuram took photographs across the day for nine consecutive days from August 28 to September 5, 2020. This helped cover a range of days with varying levels of crowding in public places as these dates overlapped with the public holidays marking the Onam festival season (official state festival of Kerala). We took photos from a range of relevant locations including public places, hospital premises, indoor gathering locations etc. Two authors independently checked all the photos to select those with a clear view of faces to confirm if they were wearing a mask. We defined proper use of mask as adequate coverage of the nose and mouth. We identified three types of masks namely cloth mask, surgical mask and N95. We used the average number of people per photo frame as a measure of crowding. We excluded photos with inadequate resolution. Children who seemed to be below 12 years of age on visual inspection were not included in the analysis.

Further, two other authors independently analyzed a random sample of the selected photos to verify the recordings. Any discordance was settled by a referee. We divided the 14 districts into three zones based on the cumulative number of COVID-19 cases reported as of 28 August 2020 as follows: High zone (districts with total cases $>$ 8000), Mid Zone (Cases 4000-8000) and Low Zone (Cases < 4000). We retrieved data on COVID-19 cases per million population for the districts from the official web portal of the government of Kerala [4]. In addition, we also analyzed the mask wearing practice as captured by the Closedcircuit television (CCTV) footage of shoppers from a shopping mall in one of the districts with a high COVID 19 burden number of cases. In this mall, COVID-19 protocols to ensure mask usage was supervised by security personnel at the entrance as well as inside the premises. We used this sample as a comparison group to study the mask usage in a supervised environment. Institutional Ethics Committee of Ananthapuri Hospitals and Research Institute, Thiruvananthapuram gave ethical clearance for the study.

\section{Results}

We took 1445 photos capturing 6208 people from all the 14 districts of Kerala. We included 4500 (73\%) people in the analysis 
as the rest of the faces were not clear enough for analysis. Of them, 1841 (52\%) were from the low zone, 1342 (30\%) from the mid zone, and 1317 (29\%) from the high zone. Half (50\%) of the photos

Table 1: Characteristics of photo samples. were taken from roadside, and around 56\% were from urban areas. Sample had 1026 (22.8\%) women. Characteristics of the photo samples are presented in Table 1.

\begin{tabular}{|c|c|c|c|c|c|c|c|c|}
\hline & Total Photos & $\begin{array}{l}\text { Total faces } \\
\text { in photos }\end{array}$ & $\begin{array}{c}\text { Average } \\
\text { Number of } \\
\text { faces per } \\
\text { photo }\end{array}$ & $\begin{array}{l}\text { Number of } \\
\text { clear faces } \\
\text { analyzed }\end{array}$ & $\begin{array}{l}\text { People with } \\
\text { mask }\end{array}$ & $\begin{array}{c}\text { Mask usage } \\
(\%)\end{array}$ & $\begin{array}{l}\text { Proper use of } \\
\text { mask }(\%)^{\#}\end{array}$ & $\begin{array}{c}\text { No mask or } \\
\text { improper } \\
\text { mask usage } \\
(\%)\end{array}$ \\
\hline Low Zone & 755 & 2448 & 3.2 & 1841 & 1765 & 95.9 & 80.8 & 22.5 \\
\hline Kannur & 100 & 381 & 3.8 & 292 & 275 & 94.2 & 87.6 & 17.5 \\
\hline Trissur & 83 & 367 & 4.4 & 255 & 243 & 95.3 & 85.2 & 18.8 \\
\hline Wayanad & 34 & 268 & 7.9 & 222 & 211 & 95 & 79.6 & 24.3 \\
\hline Idukki & 115 & 323 & 2.8 & 255 & 242 & 94.9 & 65.7 & 37.6 \\
\hline Kottayam & 162 & 422 & 2.6 & 341 & 329 & 96.5 & 86.0 & 17.0 \\
\hline Pathanamthitta & 146 & 403 & 2.8 & 251 & 241 & 96 & 82.2 & 21.1 \\
\hline Kollam & 115 & 284 & 2.5 & 225 & 224 & 99.6 & 75.9 & 24.4 \\
\hline Medium Zone & 395 & 1997 & 5.1 & 1342 & 1268 & 94.5 & 84.9 & 19.7 \\
\hline Kasaragod & 110 & 343 & 3.1 & 252 & 226 & 89.7 & 79.2 & 29.0 \\
\hline Kozhikode & 90 & 398 & 4.4 & 311 & 298 & 95.8 & 83.2 & 20.3 \\
\hline Palakkad & 43 & 252 & 5.9 & 204 & 189 & 92.6 & 92.1 & 14.7 \\
\hline Ernakulum & 100 & 697 & 7.0 & 369 & 365 & 98.9 & 83.8 & 17.1 \\
\hline Alappuzha & 52 & 307 & 5.9 & 206 & 190 & 92.2 & 89.5 & 17.5 \\
\hline High Zone & 295 & 1763 & 6.0 & 1317 & 1297 & 98.5 & 86.9 & 14.4 \\
\hline Malappuram & 62 & 300 & 4.8 & 239 & 232 & 97.1 & 88.4 & 14.2 \\
\hline Trivandrum & 233 & 1463 & 6.3 & 1078 & 1065 & 98.8 & 86.6 & 14.5 \\
\hline Kerala & 1445 & 6208 & 4.3 & 4500 & 4330 & 96.2 & 83.8 & 20.6 \\
\hline
\end{tabular}

\section{Mask Usage}

Overall, we found very high mask use, with $96 \%$ people using masks, with no difference between men and women or between urban and rural areas. We found near complete mask use in the districts with high COVID-19 burden (99\%) compared to districts with low (96\%) and the medium burden (95\%). There was statistically significant variation $(\mathrm{p}<0.001)$ between the zones. The mask usage ranged from $99.6 \%$ in Kollam district to $89.7 \%$ in Kasaragod district. We found higher mask use outdoors (96\%) than indoors (91\%). We did not find differences between hospital premises (98\%), political gatherings (98\%), or religious $(100 \%)$ gatherings. We identified the type of masks in $96 \%$ of cases. Majority (87\%) used clothes, 10\% used surgical masks and 3\% used N95 masks. We found higher use of surgical masks in hospital premises (20\%) compared to other public places (9\%); while N95 use was similar. Of those using masks ( $\mathrm{N}=4330), 84 \%$ used it properly (men $82 \%$, women $90 \%$ ).

Proper mask use was high in urban areas (86\%) than in rural $(82 \%)$; and high in the high burden zone $(86 \%)$ compared to medium (85\%) and low burden zones (82\%). We found relatively lower proportion of people wearing masks properly in public places (84\%) compared to hospital premises (95\%). Of all the mask users, nine percent did not cover their nose (men 9\%, women 7\%). This ranged from four percent in hospitals to nine percent in public places. Eight percent of mask users did not cover their nose and mouth (men 9\%, women 3\%). This ranged from four percent in hospital premises, to seven percent in other public places. There was significant difference in improper mask usage between zones with highest proportion in low zone followed by mid zone and high zone $(p<0.05)$. The analysis of data on 1059 people $(63 \%$ men) at the supervised mall showed 100\% mask use. Almost all (99.4 \%) wore their mask properly and majority of them (87\%) were cloth mask.

\section{Crowding and Social Distancing}

The average number of faces per photo frame was 4.3 for the whole state, ranging from 2.5 per frame in Kollam to 7.9 in Wayanad. Overall, the number was significantly higher in photos from high zone (6) indicating crowding in public places in districts with high COVID-19 burden. Medium zone (5.1) and low zone (3.2) had relatively low crowding (Figure 1). The total cases of COVID-19 in the districts varied from 3,643 cases in Wayanad to 34,481 in Thiruvananthapuram, as on September 30, 2020. Cases per million population varied from 3,292 in Idukki to 10,055 in Thiruvananthapuram.4 We found a weak positive correlation between crowding and cases/million population (Figure 2). 


\begin{tabular}{lrrrr|}
\hline District & Crowding & \multicolumn{2}{c|}{ Proper mask use } & COVID 19 cases \\
\hline Wayanad & $7.9 \bigcirc$ & 75.7 & 3643 \\
\hline Idukki & 2.80 & 62.4 & 3792 \\
\hline Pathanamthitta & 2.8 & 78.9 & 7845 \\
\hline Kottayam & $2.6 \bigcirc$ & 83 & 10717 \\
\hline Kasaragodu & 3.10 & 71 & 10785 \\
\hline Kannur & $3.8 \bigcirc$ & 82.5 & 11489 \\
\hline Palakkad & $5.9 \bigcirc$ & 85.3 & 11863 \\
\hline Trissur & $4.4 \bigcirc$ & 81.2 & 13633 \\
\hline Kollam & $2.5 \bigcirc$ & 75.6 & 14003 \\
\hline Alappuzha & $5.9 \bigcirc$ & 82.5 & 14261 \\
\hline Ernakulum & $7 \bigcirc$ & 82.9 & 18232 \\
\hline Kozhikode & $4.4 \bigcirc$ & 79.7 & 18819 \\
\hline Malappuram & $4.8 \bigcirc$ & 85.8 & 22543 \\
\hline Thiruvananthapuram & 6.30 & 85.5 & 34481 \\
\hline
\end{tabular}

Figure 1.

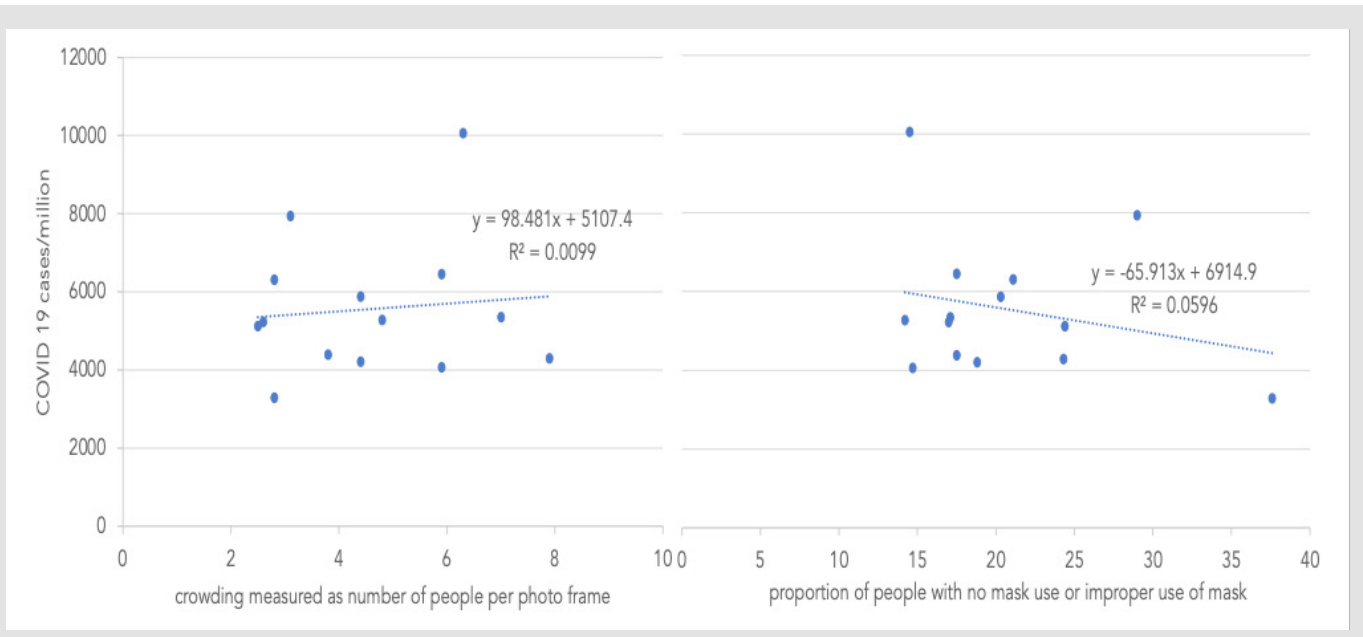

Figure 2.

\section{Discussion}

This study is the first comprehensive report on the prevalence of face mask use in any Indian state during the COVID-19 pandemic. A high prevalence of face mask usage (96\%) was found across all districts in the Kerala State, but a considerable number of people (20\%) were unprotected due to improper or non-usage of masks. The districts in high zone with higher reported cases in-fact had greater compliance to face mask usage, likely indicating the possibility that the public were more cautious due to the higher infection spread in these districts as against a possibility of low mask usage as a contributory factor for higher disease spread. A survey of self-reported mask usage from 15 countries in April 2020 reported 76\% mask usage in India, 76-91\% in Asia and 16-34\% in Europe and 50\% in USA [16,17]. Kerala has the highest number of non-resident nationals [18] and the State received the highest number of repatriation flights $(25 \%$ of the total repatriation flights by Government of India) as part of the Vande Bharat Mission [19]. This along with high interstate travel and being the 4th most densely populated state in the country puts Kerala at very high risk of import of cases as well as transmission within the state.

Despite these factors, the state of Kerala was 13th in terms of COVID-19 prevalence compared to other Indian states in the months leading up to the study period which may be attributable to the higher compliance to face mask usage in addition to many other measures [20]. In the weeks following the study, there was a surge in cases in the Kerala state with daily cases reportedly crossing 10,000 with the state climbing to 7 th position in terms of cumulative cases, 3rd position in terms of active cases and topping the charts with highest new cases per million population across the country on certain days. This could be due to several factors of which inadequate masking even in a smaller proportion of the population combined with lack of social distancing could have contributed. Previous study models have suggested that even 
$80 \%$ compliance to facemask usage in public could bring about a considerable reduction in COVID-19 transmission [19,21]. The high usage of masks in Kerala may be due to several factors including a higher literacy level and health awareness amongst the public, a robust primary health system, the "Break the Chain" campaign supported by the media as well as the political commitment of the state government. Universal masking being only one of the several aspects of disease containment, the compliance to other strategies such as hand hygiene and social distancing requires scrutiny due to the rising spread of the epidemic despite high mask compliance.

A high prevalence of mask usage was seen despite it being the Onam festive season. High compliance to face mask usage was seen across districts, even though there was a significant variation in district-wise total cases. A uniformly high usage of mask use was also seen across urban and rural areas and in various public settings. Campaigns to use masks in indoor settings had been taken up in the state to reinforce mask usage while with co-workers, friends and family indoors. But routine mask use amongst family members in residential setting may not be practical. Improper mask use was noted in $16 \%$ people with higher improper use amongst men (18\% versus $10 \%$ in women) which could be an Achilles heel to the universal mask usage campaign. The improper mask use with nose exposed versus lack of coverage of nose and mouth were equally prevalent ( $9 \%$ vs $8 \%$ ). We could identify the type of mask in $96 \%$ of people much higher that what has been previously reported by the photo-epidemiology technique.9 Cloth masks were the most commonly used masks (87\%) as is recommended for the public with a low usage of $\mathrm{N} 95$ masks (3\%) and a relatively higher usage of surgical mask in hospital premises $(20 \%$ versus $9 \%$ in other public places).

The mask usage at a shopping mall in Thiruvananthapuram was collected as a comparison group to assess mask compliance in a supervised setting. This mall has been seen to ensure strict COVID-19 protocols with the help of security personnel ensuring mask usage at the entrance as well as inside the mall. As expected, a higher prevalence of mask usage was seen in this group with 99.4\% of proper mask usage. Unabated and uncontrolled spread of COVID-19 infection suggests that mask usage alone may not be effective to prevent infection. Social distancing, hand washing, reducing the time of interaction and avoiding closed and poorly ventilated environments might be equally important to reduce infection. Average people per photo frame were used as a measure of crowding in the study. This was significantly higher in the high zone followed by medium zone and low zone. This indicates that inadequate social distancing measures could have contributed to higher cases in certain districts, despite a high compliance of mask usage across districts. The strength of the study is a relatively higher sample size representing all the districts in the State, with data collected by trained public health professionals. We could obtain only limited photos from indoor settings which limited our analysis. There is an element of subjectivity in the way photos are captured, and being a cross-sectional view, the findings reflect the behavior of people at that moment in time. Even then, the study gives a bigger picture of the mask use in the state. The crowding in photo frame as an indirect indicator of lack of social distancing may also be cautiously interpreted.

\section{Conclusion}

This cross-sectional study using photo-epidemiology method shows a high prevalence of universal mask compliance in the Indian State of Kerala during the COVID-19 pandemic. Nonetheless, the state has a surge in COVID-19 cases indicating the importance of equally emphasizing on multiple measures like social distancing, and hand hygiene, as well as the need for early diagnosis through enhanced testing.

\section{Financial Support}

None.

\section{Conflicts of Interest}

None.

\section{References}

1. Nayar KR, Koya SF, Ramakrishnan V, Rao AP, Libu GK (2020) Call to avert acceleration of COVID-19 from India's Sabarimala pilgrimage of 25 million devotees. J Travel Med 27(8): 153.

2. (2020) On Manorama Reporter. Face masks compulsory in Kerala from Thursday. On Manorama Online Newspaper.

3. (2020) Hindustan Times. Covid-19 safety guidelines mandatory for a year in Kerala; Rs10K fine for not wearing masks. Hindustan Times Newspaper (Online Version).

4. (2020) Government of Kerala. GoK Dashboard: Kerala- COVID-19 Battle.

5. Gostin LO, Cohen IG, Koplan JP (2020) Universal Masking in the United States: The Role of Mandates, Health Education, and the CDC. J Am Med Assoc 24(9): 837-838.

6. Brooks JT, Butler JC, Redfield RR (2020) Universal Masking to Prevent SARS-CoV-2 Transmission- The Time Is Now. JAMA-J Am Med Assoc 324 : 635-637.

7. (2020) Centres for Disease Control and Prevention. Considerations for Wearing Masks: Help Slow the Spread of COVID-19. Coronavirus Disease 2019 (COVID-19).

8. Patel RB, Skaria SD, Mansour MM, Smaldone GC (2016) Respiratory source control using a surgical mask: An in vitro study. J Occup Environ Hyg 13(7): 569-576.

9. Gandhi M, Yokoe DS, Havlir DV (2020) Asymptomatic Transmission, the Achilles ' Heel of Current Strategies to Control Covid-19. N Engl J Med 382(22): 2158-2160.

10. He X, Lau EHY, Wu P, Deng X, Wang J, et al. (2020) Temporal dynamics in viral shedding and transmissibility of COVID-19. Nat Med 26: 672-675.

11. Sande M Van Der, Teunis P, Sabel R (2008) Professional and HomeMade Face Masks Reduce Exposure to Respiratory Infections among the General Population. PLoS One 3(7): 3-8.

12. Macintyre CR, Hons M, Epid MA, Hasanain SJ (2020) Community universal face mask use during the COVID 19 pandemic - from households to travellers and public spaces. J Travel Med 27(3): 1-3. 
13. Chughtai AA, Seale H, Macintyre CR (2020) Effectiveness of Cloth Masks for Protection Against Severe Acute Respiratory Syndrome Coronavirus 2. Emerg Infect Dis 26(10): e200948.

14. Gandhi M, Rutherford GW (2020) Facial Masking for Covid-19- Potential for "Variolation" as We Await a Vaccine. N Engl J Med 383: e101.

15. Kumar DV (2020) How to avoid fogging up of eyeglasses while wearing masks. The New Indian Express (Newspaper). Thiruvananthapuram.

16. Elachola H, Gozzer E, Rahman NMM, Ditekemena J, Pando Robles V, et al. (2020) Photo-epidemiology to estimate face covering use in select areas in Asia versus the Americas and Africa during the COVID-19 pandemic. J Travel Med 27(8): 121.

17. Buchholz K (2020) Asians Still Most Likely to Wear Face Masks Due to COVID-19. Statista.

\section{ISSN: 2574-1241}

DOI: 10.26717/BJSTR.2021.35.005712

Shaffi Fazaludeen Koya. Biomed J Sci \& Tech Res

(C) This work is licensed under Creative

Submission Link: https://biomedres.us/submit-manuscript.php
18. (2013) Special Correspondent. State survey finds only 16.25 lakh NoRKs. The Hindu (Newspaper-Online).

19. (2020) BS Reporter. Over 500,000 Indians returned under Vande Bharat Mission, highest to Kerala. Business Standard.

20. (2020) Government of India. COVID-19 State wise Status. Covid-19 COVID19 Statewise Status.

21. Eikenberry SE, Mancuso M, Iboi E, Phan T, Eikenberry K (2020) To mask or not to mask: Modeling the potential for face mask use by the general public to curtail the COVID-19 pandemic. Infect Dis Model Elsevier Ltd 5: 293-308.

$\begin{array}{ll}\text { BIOMEDICAL } & \text { Assets of Publishing with us } \\ \text { RESEARCHES } & \text { - Global archiving of articles } \\ \text { - Immediate, unrestricted online access } & \text { - Rigorous Peer Review Process } \\ & \text { - Authors Retain Copyrights } \\ & \end{array}$

